\title{
The object as the unit of interaction between visual working memory and visual attention
}

\author{
Litian Chen, Jiewei Zheng, Mengjiao Xu, Ping Zhu, Yingtao Fu, Mowei Shen, Hui Chen \\ Department of Psychology and Behavioral Sciences, Zhejiang University, China
}

Data and materials were posted on the Open Science Framework:

https://osf.io/tphyv/

Running head: The Interaction Unit between Working Memory and Attention

\begin{abstract}
Author Note
Correspondence concerning this article should be addressed to Hui Chen or Mowei Shen, Department of Psychology and Behavioral Sciences, Zhejiang University, Xixi Campus, 148 Tianmushan Road, Hangzhou, 310007, China. Email: chenhui@zju.edu.cn or mwshen@zju.edu.cn
\end{abstract}




\begin{abstract}
This study aimed to determine the unit of interaction between visual working memory (VWM) and attention by examining two opposing hypotheses: (a) the unit of interaction is a Boolean map, a data format that can contain only one within-dimension feature (e.g., "red" or "circle"; Boolean-map-unit hypothesis) and (b) the unit of interaction is an object (object-unit hypothesis). In Experiments 1 and 2, participants held in their VWM one color, or multiple colors (two or three) that either came from one integrated object or separate objects, and then performed a visual search task that sometimes contained a distractor with a memory-matching color. Results showed that the VWMdriven attentional-capture effect triggered by colors from an integrated object was larger than that from separate objects. Moreover, surprisingly, the magnitude of the attentional guidance effect of multiple color representations from the integrated object was almost equivalent to that of a single-color object. Experiment 3 replicated the modulation effect of objecthood and demonstrated that it was not driven by different memory fidelity. Experiments 4 and 5 extended these findings by generalizing the effect to two features from different dimensions (i.e., color and orientation), and from different modules (i.e., visual and verbal color representations). These results suggest the objecthood of multiple representations could modulate their ability to guide attention, supporting the object-unit hypothesis. These findings have crucial implications for understanding the architecture of interactions between VWM and attention.
\end{abstract}

Keywords: Visual working memory, Visual attention, Attentional guidance 
There is a close interaction between visual working memory (VWM) and attention. Recently, this interaction has been demonstrated by the observation that the representations held in VWM could automatically influence attentional selection in external world, a phenomenon known as the working memory-driven attentional capture effect (van Moorselaar et al., 2014; Ort \& Olivers, 2020; Williams et al., 2019; Sasin \& Fougnie, 2020). For example, when hold a banana in mind, and look for something else among numerous products in a large grocery store, our attention would be attracted by any stimuli matching the feature of banana (e.g., yellow color) in the environment, rather than only the search product. However, when we hold a banana and a tomato in our mind at the same time, it is unclear whether our attention would be similarly captured by items containing relevant features (e.g., red or yellow color). This has generated a lively debate regarding how many items in VWM can simultaneously interact with attention.

Numerous studies have provided robust evidence of attentional guidance by a single item in VWM (e.g., Downing \& Dodds, 2004; Fu et al., 2021; Houtkamp \& Roelfsema, 2006; Olivers et al., 2006; Olivers et al., 2011; Soto et al., 2005; Soto et al., 2012). For instance, when participants were asked to hold a single feature (e.g., color) in VWM, and then perform a visual search task, their attention was captured by any stimuli with that feature, even if it was a distractor rather than a search target. However, the results have been mixed when multiple items are held in VWM, with some studies showing that attentional guidance can occur with multiple items (e.g., Bahle et al., 2019; Grubert \& Eimer, 2015; 2016; Hollingworth \& Beck, 2016; Zhou et al., 2020), while 
others have not reported such an effect even using similar paradigms (e.g., Beck \& Vickery, 2019; van Moorselaar et al., 2014; Williams et al., 2019). For example, Van Moorselaar et al. (2014) asked participants to hold a variable number (1-4) of colors in VWM and found that, while a reliable memory-driven attentional capture effect could be observed when only one item was in VWM, such effect disappeared when there was more than one item in VWM. In contrast, Hollingworth and Beck (2016) found memory-driven capture even when multiple colors were kept in VWM, and moreover, this capture effect driven by multiple representations was larger in comparison with that triggered by one single color representation in some certain contexts. Although the above debate remains unresolved yet, the attentional guidance effect with multiple items is at least relatively unreliable in comparison with a single one item, which suggests that the ability of guiding attention is impaired when participants are asked to maintain more than one item in VWM.

This debate has broader implications for understanding the architecture of VWM. The items held in VWM that could guide attention has been considered to possess an active state, known as Focus of Attention (FoA) in some working memory models (Cowan, 2001, 2005; McElree, 2006; Oberauer, 2002; Oberauer \& Bialkova, 2009). Similar with the above debate, these models have different views on the capacity of FoA. For example, Oberauer (2002) proposed that only one item could be held in the FoA state, which is consistent with the different states account that only one representation in the active state could guide attention (Olivers et al., 2011). While in Cowan's model (2005), multiple items could be in FoA and simultaneously guide 
attention (Bahle et al., 2019). Therefore, understanding the process of how VWM interact with visual attention is critical for understanding the nature of VWM architecture.

The ongoing debate regarding how many items in VWM can simultaneously interact with attention (i.e., the capacity of interaction) has focused mainly on assessing whether and when two items can simultaneously guide attention. However, when we discuss how many items in VWM can interact with attention simultaneously, we have to firstly define what an "item" is. In other words, to explore the capacity of the interaction, the unit of such interaction should be clarified. This issue is central to the above-mentioned debate, however, has been completely overlooked. Moreover, addressing the nature of interaction item/unit has broad implications for clarifying the architecture of the interaction between VWM and attention. Thus, the current study sought to directly address this issue by testing two opposing hypotheses.

First, the Boolean-map-unit hypothesis posits that the interaction unit is a Boolean map. Huang and Pashler (2007) initially proposed the Boolean-map theory as a unified framework of attentional processing. According to this theory, a Boolean map is a data format of the feature label or spatial structure, which is considered as the unit of conscious access, namely, what can be consciously perceived at any given instant (Huang, 2010; Huang et al., 2007). Any given Boolean map can only contain a single feature label per dimension (e.g., color is green; shape is a square) and thus it is impossible to simultaneously access two or more features from same dimension (e.g., a red and a green color) regardless of whether they come from the same or different 
objects. Moreover, Huang (2020) recently extended this theory from visual attention to VWM by demonstrating that the unit of VWM storage is a Boolean map rather than an object. Thus, it is reasonable to speculate that the unit of VWM representations interacting with attention is a Boolean map as well. Accordingly, when people held two different colors in mind, those two colors would be represented as two distinct Boolean maps in VWM, regardless of whether they belong to an integrated object or two separate ones. Thus, there should be always two Boolean maps interacting with attention, resulting the same attentional guidance effect of multiple representations no matter when they come from one object or not.

Second, the object-unit hypothesis proposes that the interaction unit between VWM and attention is an object rather than a Boolean map. As mentioned by Huang (2020), perceptual structure (e.g., object) plays an important role in information storage within the VWM, despite the unit of VWM storage being a Boolean map. Thus, it is possible that multiple Boolean maps might be grouped together in VWM, particularly if encoded from an integrated object (e.g., a circle comprising two different-colored semicircles), and thereby interact with attention as a coherent object. In other words, the unit of interaction might be an object consisting of multiple Boolean maps. According to this hypothesis, the objecthood of multiple representations should play a critical role in driving attentional guidance. Specifically, when people held two colors from an integrated object in VWM, they should be able to interact with attention as one single unit. However, when the two colors came from two separate objects, the interaction process would be affected because there are two units interacting with 
attention in this case. Therefore, we should observe a significant difference of attentional capture effect when two colors come from two objects compared with when they come from one single object.

To differentiate between these two hypotheses, the current study adopted the classical VWM-driven attentional capture paradigm wherein participants held some contents (e.g., color) in their VWM, and then performed a visual search task that could sometimes include a distractor with memory-matching information. Importantly, we manipulated the perceptual organization (objecthood) of the memorized color representations (e.g., red and green), specifically, whether two different colors came from two separate objects or an integrated object. If the Boolean-map-unit hypothesis is correct, there are always two Boolean maps simultaneously interacting with attention, regardless of whether the two colors are encoded from the same or different objects. Thus, VWM-driven attentional guidance should not be influenced by the objecthood of two colors. Otherwise, if the object-unit hypothesis is true, we should observe a substantial impact of objecthood on the attentional capture effect, which should be stronger when two different colors from an integrated object (wherein only a unit/object interacts with attention) than when two colors from two separate objects (wherein two units/objects interact with attention).

\section{Experiment 1}

The first experiment assessed whether attentional capture would be influenced by the objecthood of multiple representations in VWM, which was manipulated by 
presenting two different colors either in a perceptually integrated object, or two separate objects.

\section{Methods}

\section{Participants}

Sixty native Chinese students from Zhejiang University, randomly divided into three groups, completed Experiment 1 for either a course credit or 15 RMB. Sample size (20) was estimated based on a medium effect size $\left(\eta^{2}=.40\right)$ from previous studies using a similar paradigm (e.g., Olivers, 2009; Sun et al., 2015), and maintained constant across experiments. All participants provided informed consent, and all procedures were approved by the Institutional Review Board at Zhejiang University.

\section{Stimuli}

All stimuli were displayed on a white background ( $\mathrm{RGB}$ values $=255,255$, 255). As depicted in Figure 1, in the separate group, the memory items were two separated colored semicircles (with a radius of $2.01^{\circ}$ ), separated (center to center) $0.57^{\circ}$ vertically and $1.01^{\circ}$ horizontally. In the integrated group, there was a circle composed of two differently colored semicircles. In the single-color group, there was a singlecolor circle. The color set included five different colors (RGB values $=255,0,0$ [red]; 0, 255, 0 [green]; 0, 0, 255 [blue]; 255, 255, 0 [yellow] and 255 0, 255 [purple]).

Each search display consisted of eight disks $\left(2.86^{\circ}\right)$ equally distributed on an imaginary circle $\left(\right.$ radius $\left.=6.87^{\circ}\right)$ centered on a fixation cross. One of these disks was defined as the target, which included a single $45^{\circ}$ tilted line (left or right). The others were gray $(\mathrm{RGB}=192,192,192)$ distractor disks which contained a black $(\mathrm{RGB}=0$, 
$0,0)$ "X". In each group, the test display had the same layout as the memory display.

\section{Procedure and design}

As shown in Figure 1, each trial started with a 500 ms fixation display, followed by an $800 \mathrm{~ms}$ memory display. In the integrated and separate groups participants were instructed to memorize both colors from the two-colored semicircles, while in the single-color group they were asked to memorize the circle's only color. After a $180 \mathrm{~ms}$ blank screen, the search display appeared until response or $3000 \mathrm{~ms}$ had elapsed. Participants had to search for the target disk among seven distractor disks, and indicate whether the line inside the target disk tilted left or right as quickly and accurately as possible, by pressing the corresponding left or right arrow key with their right hands. In $2 / 3$ of the trials, the search display contained a color singleton distractor, which either had a color matching the color representation in memory (i.e., color match condition) or an unrelated color (i.e., mismatch condition). In the remaining $1 / 3$ no-singleton distractor trials, all distractors were gray.

After participants completed the search task, a blank screen appeared for 500 $\mathrm{ms}$, and then a test display appeared until response or $4000 \mathrm{~ms}$ had elapsed. The test item had the same colors as those in the memory display in half the trials and different ones in the other half. Participants had to indicate whether the two colors in the test display were identical to the memory display, by pressing " $\mathrm{J}$ " if they were identical or " $\mathrm{K}$ " if they differed with their left hands. If different, only one of the semicircles changed its color.

Participants in each group completed a practice block of 8 trials and 4 
experimental blocks of 60 trials each: 20 color match trials, 20 mismatch trials, and 20 no-singleton trials.

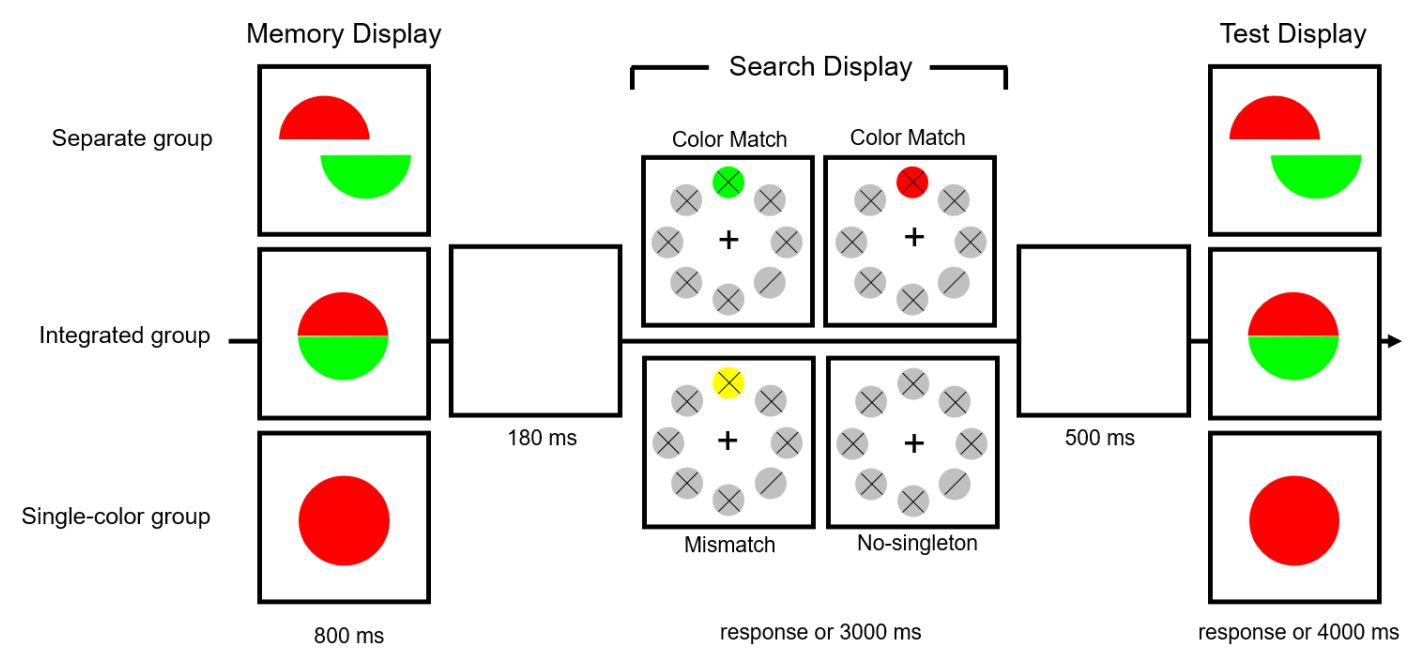

Figure 1. Example sequences of a trial in Experiment 1.

\section{Data analysis}

Trials with Reaction times (RTs) over 2.5 standard deviations on the search task $(2.6 \%)$ or incorrect on either the search or memory task $(3.82 \%)$ were excluded from analysis. The memory-driven attentional capture effect was calculated as the RT difference between the color match and mismatch conditions on the search task. The accuracy of the memory task in this experiment, and in following experiments, was close to ceiling, and did not differ between the integrated and separate groups (see the detailed accuracy data in the supplementary materials).

\section{Results}

As shown in Figure 2, there was a reliable memory-driven attentional capture effect in both the integrated group (color match vs. mismatch: 794 ms vs. 775 ms, Mean Difference $[\mathrm{MD}]=19 \mathrm{~ms}, 95 \%$ CI $[6.72,31.88], t(19)=3.212, p=.005$, Cohen's $d=$ 
0.718) and the single-color group (color match vs. mismatch: $782 \mathrm{~ms}$ vs. $756 \mathrm{~ms}$, $\mathrm{MD}=26 \mathrm{~ms}, 95 \% \mathrm{CI}[17,19,37.16],(19)=4.987, p<.001$, Cohen's $d=1.115)$. However, the attentional capture effect was completely absent in the separate group (color match vs. mismatch: $774 \mathrm{~ms}$ vs. $775 \mathrm{~ms}, \mathrm{MD}=-1 \mathrm{~ms}, 95 \% \mathrm{CI}[-12.29,10.41], t$ $(19)=-.174, p=.864$, Cohen's $\left.d=-0.039, \mathrm{BF}_{10}=0.236\right)$.

More importantly, the between-group comparison showed that the attentionalcapture effect of colors significantly differed between the separate and the integrated groups $\left(F(1,38)=6.256, p=0.017, \eta_{p}^{2}=0.141\right)$, and between the separate group and the single-color group $\left(F(1,38)=12.914, p=0.001, \eta_{p}{ }^{2}=0.208\right)$. However, there was no significant difference between the integrated and single-color groups $(F(1,38)=$ $\left.0.741, p=0.395, \eta_{p}^{2}=0.019, \mathrm{BF}_{10}=0.441\right)$

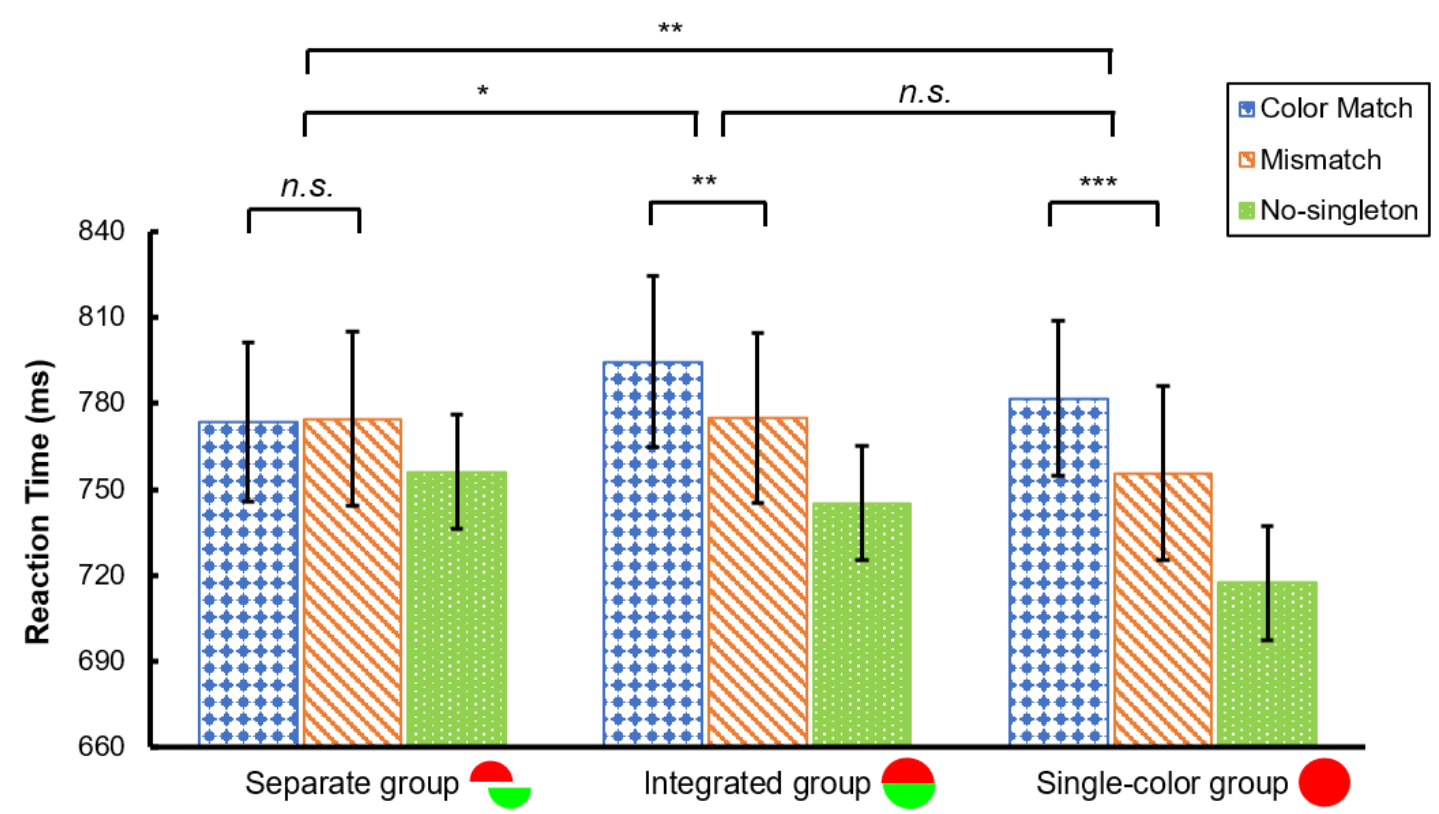

Figure 2. Mean search RT results for Experiment 1. * $p<.05 . * * p<.01$. *** $p$ $<.001$. n.s. $=$ not significant. Error bars represent standard error of the mean (SEM).

Experiment 1 showed that two different color representations in VWM could simultaneously capture attention when encoded from an integrated object, but not when 
encoded from two separate objects. Importantly, this WM-driven attentional capture effect triggered by the color from the integrated object was significantly larger than that from the separate object. These findings demonstrated that the objecthood of multiple representations in VWM could significantly modulate their ability to interact with attention, directly supporting the object-unit hypothesis. Moreover, the magnitude of the attentional capture effect elicited by the color from the integrated object was nearly equivalent to that from the single-color object, which suggests that two different colors from a perceptually integrated object could interact with attention just as well as a single one.

\section{Experiment 2}

The current experiment sought to further test the object-unit hypothesis by investigating whether the results of Experiment 1 could be replicated and generalized to a case wherein participants have to hold more than two colors in VWM (e.g., three different colors).

\section{Methods}

This experiment was identical to Experiment 1 except for the following: Forty native Chinese students from Zhejiang University completed Experiment 2. As depicted in Figure 3, in the integrated group, the memory display contained a circle made up of three equal sectors with different colors, while in the separate group the three equal sectors changed as follows: two sectors moved $1.05^{\circ}$ and $1.46^{\circ}$ outward along the centerline, then they were rotated $40^{\circ}$ and $20^{\circ}$ in the same direction (clockwise or counterclockwise), respectively; while another sector moved $5.34^{\circ}$ outward along 
the centerline and flipped $180^{\circ}$. The colors used in this experiment contained the same color set as in Experiment 1 plus a new color (RGB values = 0,255,255 [Cyan]).

Participants in each group completed a practice block of 8 trials and 3 experimental blocks of 90 trials each: 30 color match trials, 30 mismatch trials, and 30 no-singleton trials.

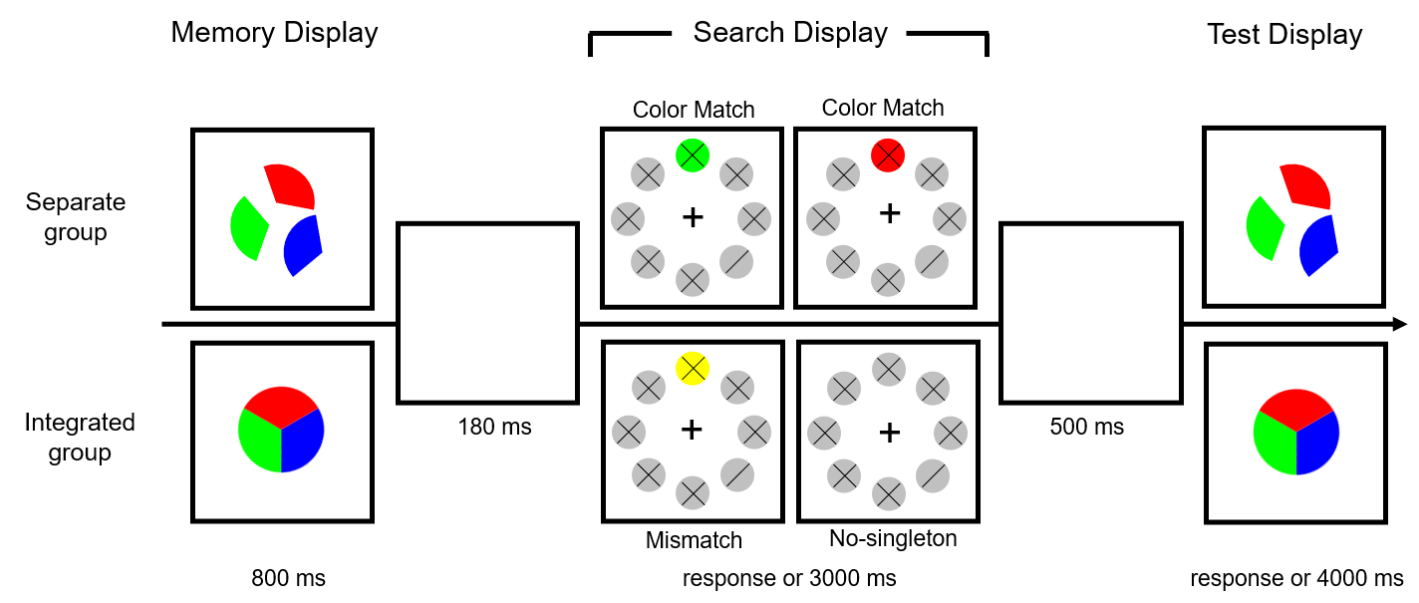

Figure 3. Example sequences of a trial in Experiment 2.

\section{Results}

Data analysis was the same as Experiment 1; thus, $2.66 \%$ and $8.39 \%$ of trials were excluded from analysis due to RTs outliers and response errors, respectively. As shown in Figure 4, there was a reliable memory-driven attentional capture effect in the integrated group (color match vs. mismatch: $779 \mathrm{~ms}$ vs. $763 \mathrm{~ms}, \mathrm{MD}=16 \mathrm{~ms}$, 95\% CI $[4.40,28.46], t(19)=2.858, p=.010$, Cohen's $d=0.639)$, but the capture effect was completely absent in the separate group (color match vs. mismatch: $765 \mathrm{~ms}$ vs. $769 \mathrm{~ms}$, $\mathrm{MD}=-4 \mathrm{~ms}, 95 \% \mathrm{CI}[-14.20,6.93], t(19)=-.721, p=.480$, Cohen's $d=-0.161, \mathrm{BF}_{10}=$ 0.293). Importantly, between-group comparisons showed that the attentional-capture effect of the color significantly differed between the separate and integrated group, as 
indicated by a significant interaction effect between the two groups $(F(1,38)=6.880$, $\left.p=0.012, \eta_{p}{ }^{2}=0.153\right)$. Furthermore, we compared the attentional-capture effect from the integrated group of the current experiment with that from the single-color group of Experiment 1 and found no significant difference between these two groups $(F(1,38)$ $\left.=1.568, p=0.218, \eta_{p}^{2}=0.040, \mathrm{BF}_{10}=0.513\right)$.

Consistent with Experiment 1, the results of Experiment 2 demonstrated that the objecthood of VWM representations significantly influences their ability for attentional guidance, even in case of three color representations simultaneously interacting with attention. Moreover, the attentional guidance was comparable between one integrated object consisting of three different colors and one object with a single color. Together, these findings provide stronger evidence supporting the object-unit hypothesis.

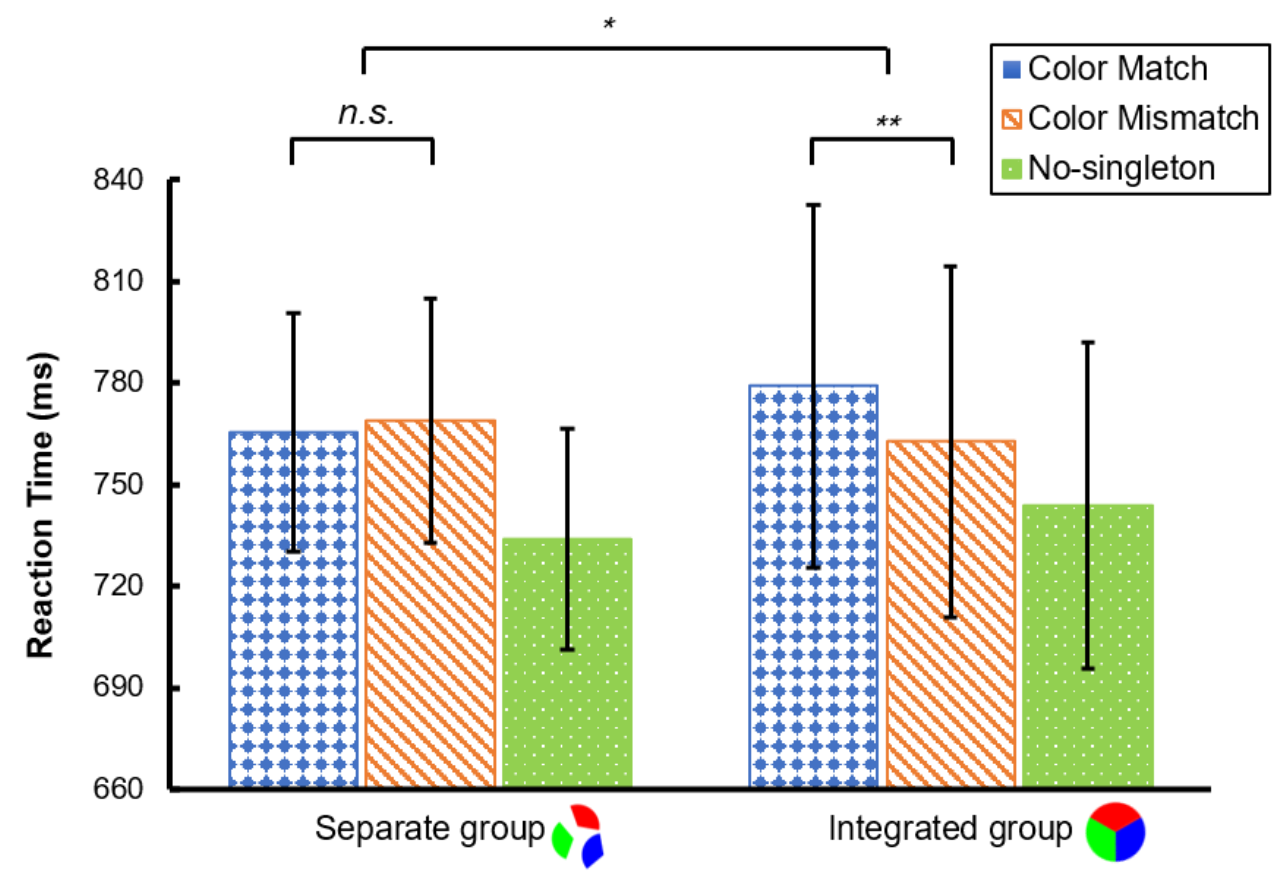

Figure 4. Mean search RT results for Experiment 2. * $p<.05 . * * p<.01$. *** $p$ $<.001$. n.s. $=$ not significant. Error bars represent SEM. 


\section{Experiment 3}

The first aim of this experiment was to demonstrate the observed effect of objecthood in previous experiments was robust by replicating Experiment 1 with a larger sample size. Moreover, this experiment sought to rule out an alternative explanation that the differences in capture between the integrated and separate group was driven by the differences in memory fidelity in these two conditions, as a recent study found that the representational fidelity of memories would influence their attentional guidance (Williams et al., 2019). Thus, we used a more sensitive memory test (i.e., a color wheel task that could measure precision), instead of change detection task as in previous experiments.

\section{Methods}

Forty native Chinese students in each group from Zhejiang University completed this experiment. The experimental procedure was identical to Experiment 1 except for the following: As shown in Figure 5, during the test display, a color wheel with a radius of $4^{\circ}$ and thickness of $0.5^{\circ}$, appeared around memory items in the center of the screen. The color wheel was composed of 360 colored segments. One of the test items had the same colors as that in the memory display, another was white with visible boundaries. Participants were asked to make a response regarding the color of the white item by clicking on the color wheel, then press the down arrow key with their right hands to confirm their response. 


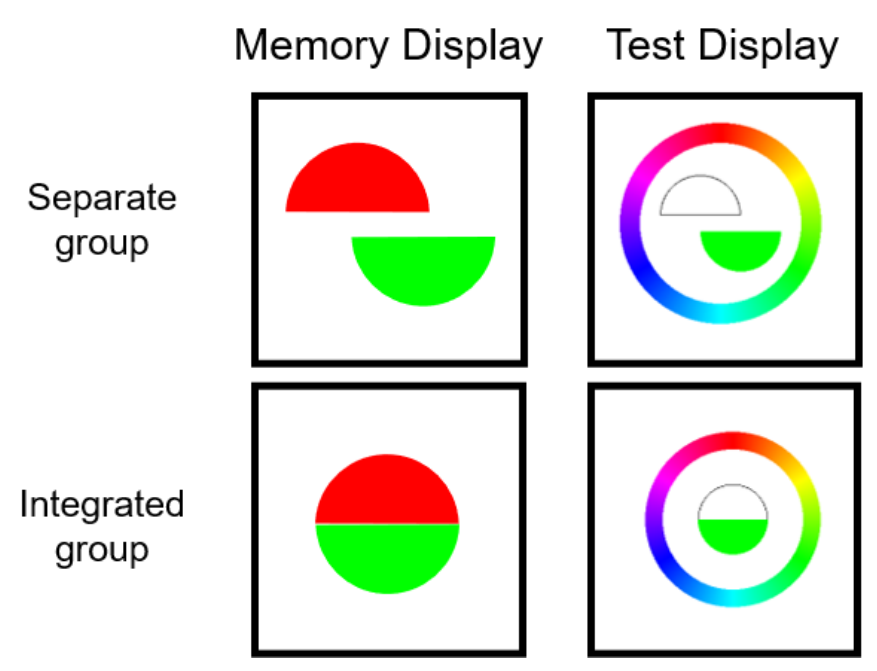

Figure 5. Example of memory display and test display in Experiment 3.

\section{Results}

Data analysis was the same as previous experiments. Trials with RTs over 2.5 standard deviations on the search task $(2.77 \%)$ or incorrect on the search task $(1.43 \%)$ were excluded from search RT analysis.

As shown in Figure 6A, there was a reliable memory-driven attentional capture effect in the integrated group (color match vs. mismatch: $858 \mathrm{~ms}$ vs. $828 \mathrm{~ms}$, $[\mathrm{MD}]=30 \mathrm{~ms}, 95 \%$ CI $[19.01,40.526], t(39)=5.597, p<.001$, Cohen's $\mathrm{d}=0.885)$. Whereas the attentional capture effect in the separate group did not reach significance (color match vs. mismatch: 819 ms vs. $811 \mathrm{~ms}, \mathrm{MD}=7 \mathrm{~ms}, 95 \% \mathrm{CI}[-2.14,17.13]$, t (39) $=1.573, p=.124$, Cohen's $\mathrm{d}=0.249, \mathrm{BF}_{10}=0.529$ ). More importantly, the betweengroup comparison showed that the attentional-capture effect of colors was significantly larger in the integrated group than in the separate group $(F(1,78)=9.732, p=0.003$, $\left.\eta_{p}^{2}=0.111\right)$

The memory error was defined as the absolute deviation in the degree distance on the color wheel between the memory report color and true value. As shown in Figure 
$6 \mathrm{~B}$, there was no significant difference in the mean errors of memory fidelity between the two groups $(5.58$ vs. $5.96, \mathrm{MD}=-0.38,95 \%$ CI $[-1.693, .942], t(78)=-.567, p=$ 0.572, Cohen's $\left.\mathrm{d}=-0.127, \mathrm{BF}_{10}=0.267\right)$.

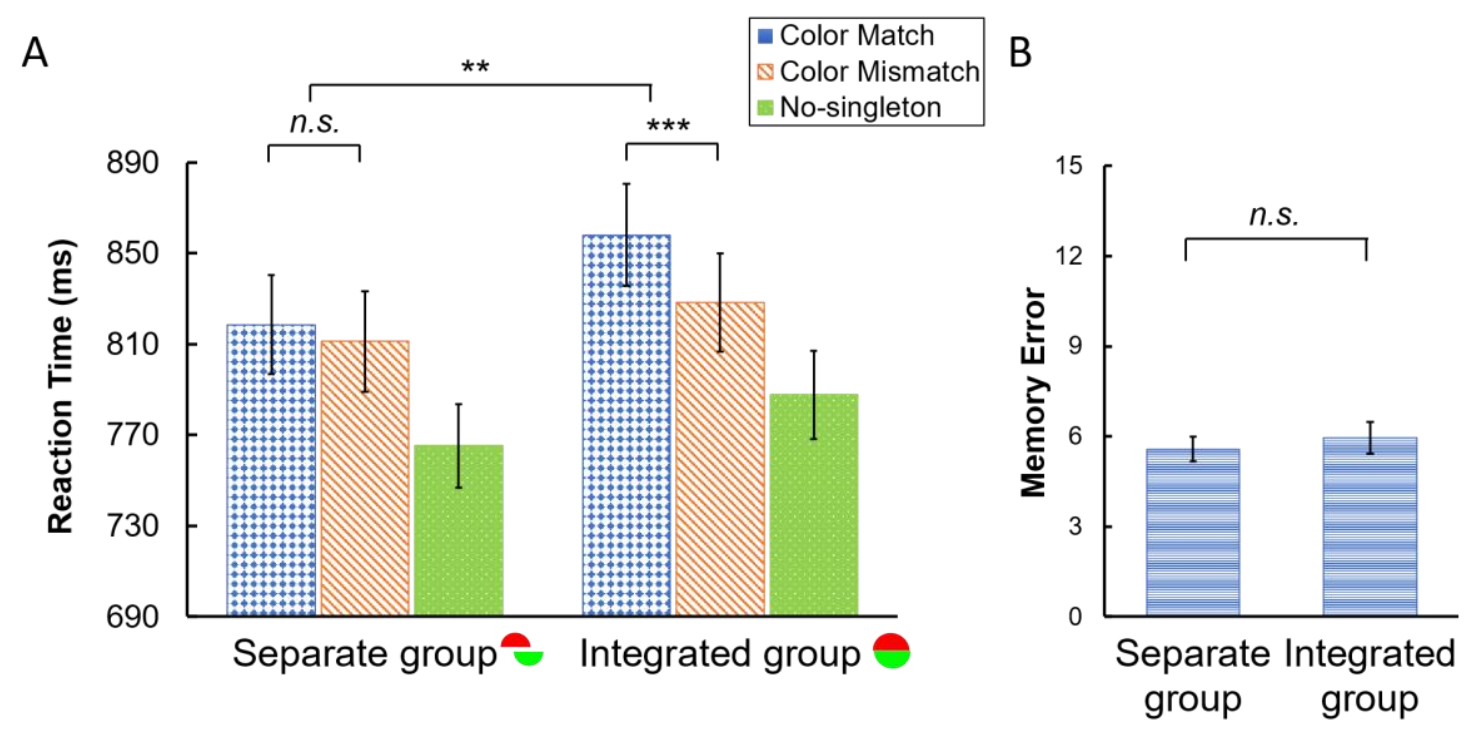

Figure 6. (A) Mean search RT results for Experiment 3. (B) Mean memory errors for Experiment 3. $* p<.05$. ** $p<.01$. *** $p<.001$. n.s. = not significant. Error bars represent SEM.

As expected, the results replicated the effect of objecthood shown in Experiment 1 even when the sample size was doubled. Importantly, this experiment suggested that this effect was not driven by the difference of memory fidelity, as no significant difference was found between the integrated and separate groups.

\section{Experiment 4}

Previous experiments showed a reliable objecthood effect for features from same dimension (i.e., colors). This experiment sought to further explore whether the findings could be generalized to two features from different dimensions (i.e., color and orientation).

\section{Methods}


Forty native Chinese students in each group from Zhejiang University completed Experiment 4. The experiment was identical to Experiment 1 except as follows: As shown in Figure 7, we used the classical mushroom-like stimuli as in previous studies (Xu, 2002), which combined orientation and color information. The mushroom stems were black and oriented to either left or right. The cap colors were identical as in Experiment 1. Participants were asked to remember both the cap color and stem orientation. The cap and stem of the mushroom in the memory display were attached for the integrated group, while they were detached for the separate group.

Since there was orientation information in the memory task, we replaced the orientation judgement task in the search display used in previous experiments to avoid any confusions. Instead, participants were asked to search for the target diamond and indicate whether the letter inside it was an $\mathrm{M}$ or $\mathrm{N}$ as quickly and accurately as possible. There were five different conditions in the search display. In the color match condition, the singleton distractor had a color matching the cap color held in memory. In the color mismatch condition, the singleton color was an unrelated color. In the orientation match condition, the singleton distractor was a mushroom stem with the same orientation as the memory item. In the orientation mismatch condition, the orientation of the singleton stem was different from the memory item. In the no-singleton condition, all the distractors were gray disks.

Participants in each group completed a practice block of 8 trials and 4 experimental blocks of 60 trials each: 10 color match trials, 10 color mismatch trials, 10 orientation match trials, 10 orientation mismatch trials, and 20 no-singleton trials. 


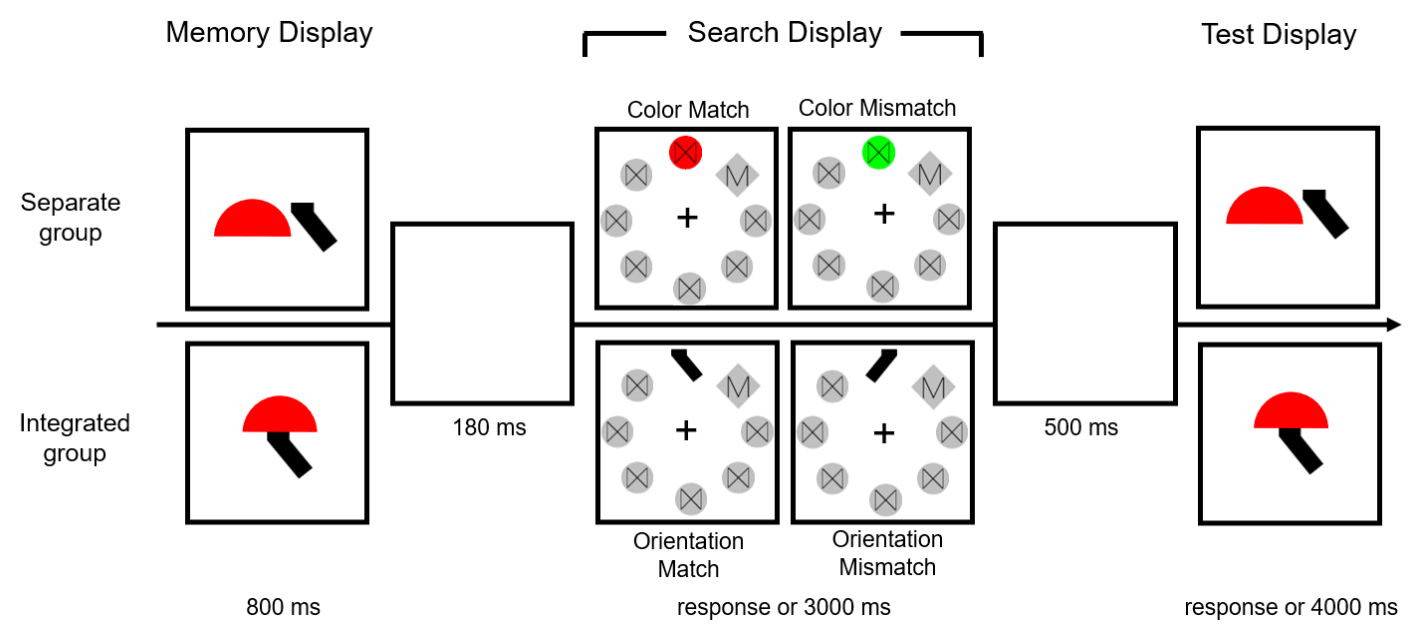

Figure 7. Example sequences of a trial in Experiment 4.

\section{Results}

Data analysis was the same as previous experiments; Trials with RTs over 2.5 standard deviations on the search task $(2.04 \%)$ or incorrect on either the search or memory task $(5.68 \%)$ were excluded from analysis. The results were shown in Figure 8. For the color dimension, there was a reliable memory-driven attentional capture effect in the integrated group (color match vs. color mismatch: $1152 \mathrm{~ms}$ vs. $1124 \mathrm{~ms}$, $\mathrm{MD}=28 \mathrm{~ms}, 95 \%$ CI [9.20, 46.07], $t(39)=3.032, p=.004$, Cohen's $d=0.479)$, and no capture effect was found in the separate group (color match vs. color mismatch: 1174 ms vs. $1181 \mathrm{~ms}, \mathrm{MD}=-7 \mathrm{~ms}, 95 \% \mathrm{CI}[-34.04,20.85], t(39)=-0.486, p=.630$, Cohen's $\left.d=-0.077, \mathrm{BF}_{10}=0.191\right)$. Moreover, between-group comparisons showed that there was a significant difference of attentional-capture effect between the integrated group and separate group for color dimension $\left(F(1,78)=4.385, p=0.039, \eta_{p}^{2}=0.053\right)$. However, for the orientation dimension, no significant attentional capture effect was found in either the integrated group (orientation match vs. orientation mismatch: 1123 ms vs. $1117 \mathrm{~ms}, \mathrm{MD}=6 \mathrm{~ms}, 95 \%$ CI [-18.36, 31.16],t (39) $=0.523, p=.604$, Cohen's $d$ 
$=0.083, \mathrm{BF}_{10}=0.194$ ) or separate group (orientation match vs. orientation mismatch:

$1198 \mathrm{~ms}$ vs. $1189 \mathrm{~ms}, \mathrm{MD}=9 \mathrm{~ms}, 95 \% \mathrm{CI}[-18.19,35.88], t(39)=0.667, p=.509$,

Cohen's $d=0.105, \mathrm{BF}_{10}=0.210$ ), and there was no significant difference between these two groups $\left(F(1,78)=0.019, p=0.891, \eta_{p}^{2}=0.000, \mathrm{BF}_{10}=0.214\right)$.

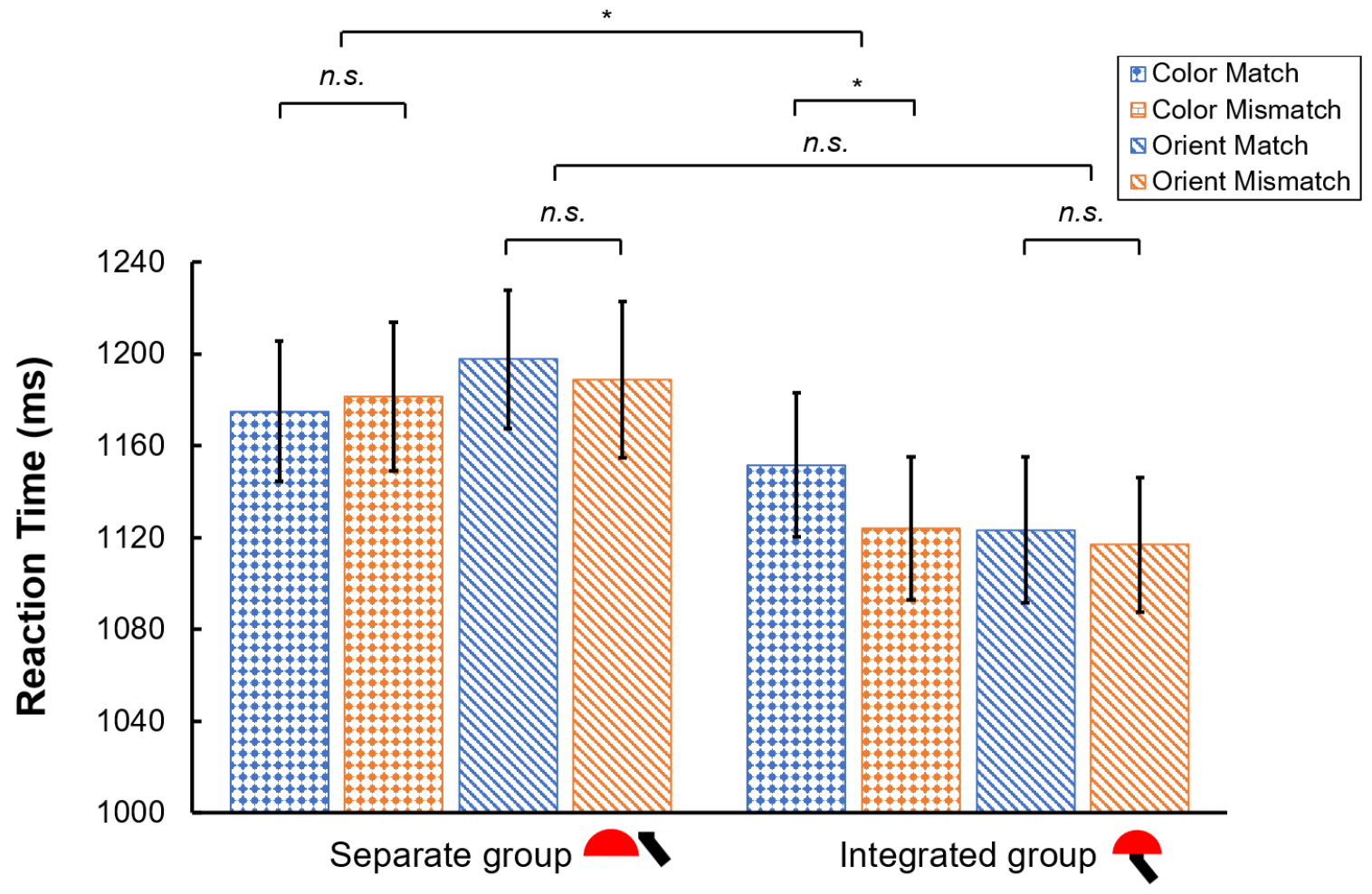

Figure 8. Mean search RT results for Experiment 4. * $p<.05 . * * p<.01$. *** $p$ $<.001$. n.s. $=$ not significant. Error bars represent SEM.

Consistent with previous experiments, these results showed a larger attentional guidance effect in the integrated group than in the separate group, indicating that the effect of objecthood on attentional guidance could be generalized to the features from different dimensions. Note that the effect was observed only for the color dimension, we even did not find any attentional guidance effect in either the integrated or separate group for the orientation, which was consistent with previous studies showing that orientation information was poor at triggering attentional guidance (Sasin \& Fougnie, 
2020).

\section{Experiment 5}

To further broaden our findings, this experiment sought to generalize our results by using two features from different modules (i.e., the ink color or identity of the Chinese characters, which corresponds to visual-spatial sketchpad and phonological loop according to the three-component model of working memory, Baddeley \& Hitch, 1974), as many previous studies showed that these cross-module color representations (i.e., visual and verbal representations) could be bound into a unitary object and stored in some domain-general episodic buffer in working memory (i.e., cross-module binding, Allen et al., 2006; Baddeley, Allen, \& Hitch 2011; Gao et al., 2017; Langerock et al., 2014).

\section{Methods}

Forty native Chinese students in each group from Zhejiang University completed this experiment. The experiment was identical to Experiment 1 except as follows. As shown in Figure 9, in the separate group, the memory display contained a colored square $\left(4.01^{\circ} \times 4.01^{\circ}\right)$ and a black Chinese character $\left(4.01^{\circ} \times 4.01^{\circ}\right)$, with identity of the Chinese character indicating a color that was different from the color of the square. Participants were instructed to memorize both the color of the square and the color indicated by the Chinese character. In the integrated group, the memory display contained a colored Chinese character, whose identity indicated a color that differed from its "ink" color. Participants were asked to remember the Chinese character's identity (i.e., what color it signified) and the actual color in which the 
character itself was presented (i.e., its "ink" color). There were five different colored squares (the colors were chosen from the same set as in Experiment 1) and five corresponding Chinese characters indicating the same colors as the squares. There were four different conditions in the visual search display. In the ink color match condition, the singleton color matched the memorized color of the square (for the separate group) or the ink color of the Chinese character (for the integrated group). In the identity match condition, the singleton color matched the identity of the Chinese character. In the mismatch condition, the singleton color did not match any of the colors held in memory. In the no-singleton condition, all the distractors on the search display were grayscale.

Participants in each group completed a practice block of 12 trials and 4 experimental blocks of 60 trials each: 10 ink-color match trials, 10 identity match trials, 20 mismatch trials, and 20 no-singleton trials.

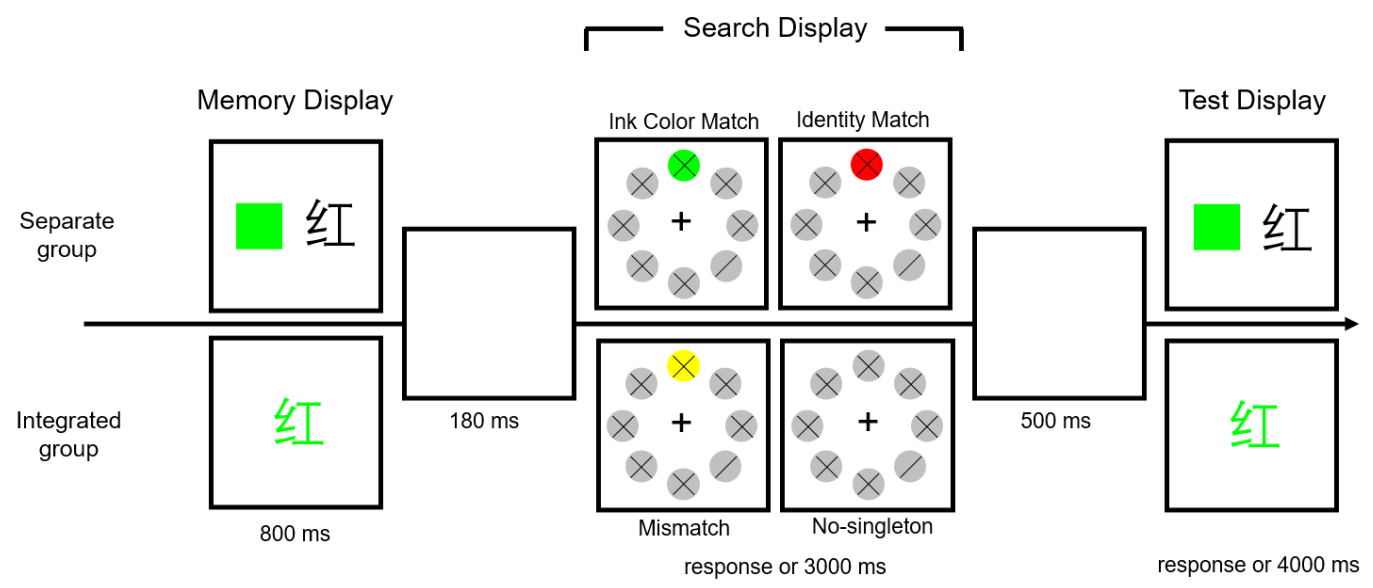

Figure 9. Example sequences of a trial in Experiment 5.

\section{Results}

Data analysis was the same as previous experiments; Trials with RTs over 2.5 standard deviations on the search task $(2.63 \%)$ or incorrect on either the search or 
memory task $(3.86 \%)$ were excluded from analysis. As shown in Figure 10, in the integrated group, there was a reliable memory-driven attentional capture effect. The RTs in both the ink color match and identity match conditions were significantly higher than the mismatch condition (ink color match vs. mismatch: $741 \mathrm{~ms}$ vs. $714 \mathrm{~ms}, \mathrm{MD}=$ $27 \mathrm{~ms}, 95 \%$ CI $[15.15,37.69], t(39)=4.741, p<.001$, Cohen's $d=0.750 ;$ identity match vs. mismatch: $733 \mathrm{~ms}$ vs. $714 \mathrm{~ms}, \mathrm{MD}=19 \mathrm{~ms}, 95 \%$ CI [5.38, 32.24], $t(39)=2.833$, $p=.007$, Cohen's $d=0.448$ ). However, in the separate group, this memory-driven attentional capture effect was absent. There was no significant difference for the RTs between the match conditions and the mismatch condition (ink color match vs. mismatch: $794 \mathrm{~ms}$ vs. $788 \mathrm{~ms}, \mathrm{MD}=6 \mathrm{~ms}, 95 \% \mathrm{CI}[-5.26,17.42], t(39)=1.084, p=.285$, Cohen's $d=0.171, \mathrm{BF}_{10}=0.295$; identity match vs. mismatch: $788 \mathrm{~ms}$ vs. $788 \mathrm{~ms}, \mathrm{MD}=$ $0 \mathrm{~ms}, 95 \%$ CI $[-14.50,13.77], t(39)=-.052, p=.959$, Cohen's $\left.d=-.008, \mathrm{BF}_{10}=0.171\right)$. Furthermore, between-group comparisons showed that the memory-driven attentional capture effect differed significantly between the separate and integrated groups both in the ink color condition $\left(F(1,78)=6.619, p=0.012, \eta_{p}{ }^{2}=0.078\right)$, and in the identity condition $\left(F(1,78)=3.956, p=0.05, \eta_{p}^{2}=0.048\right)$. 


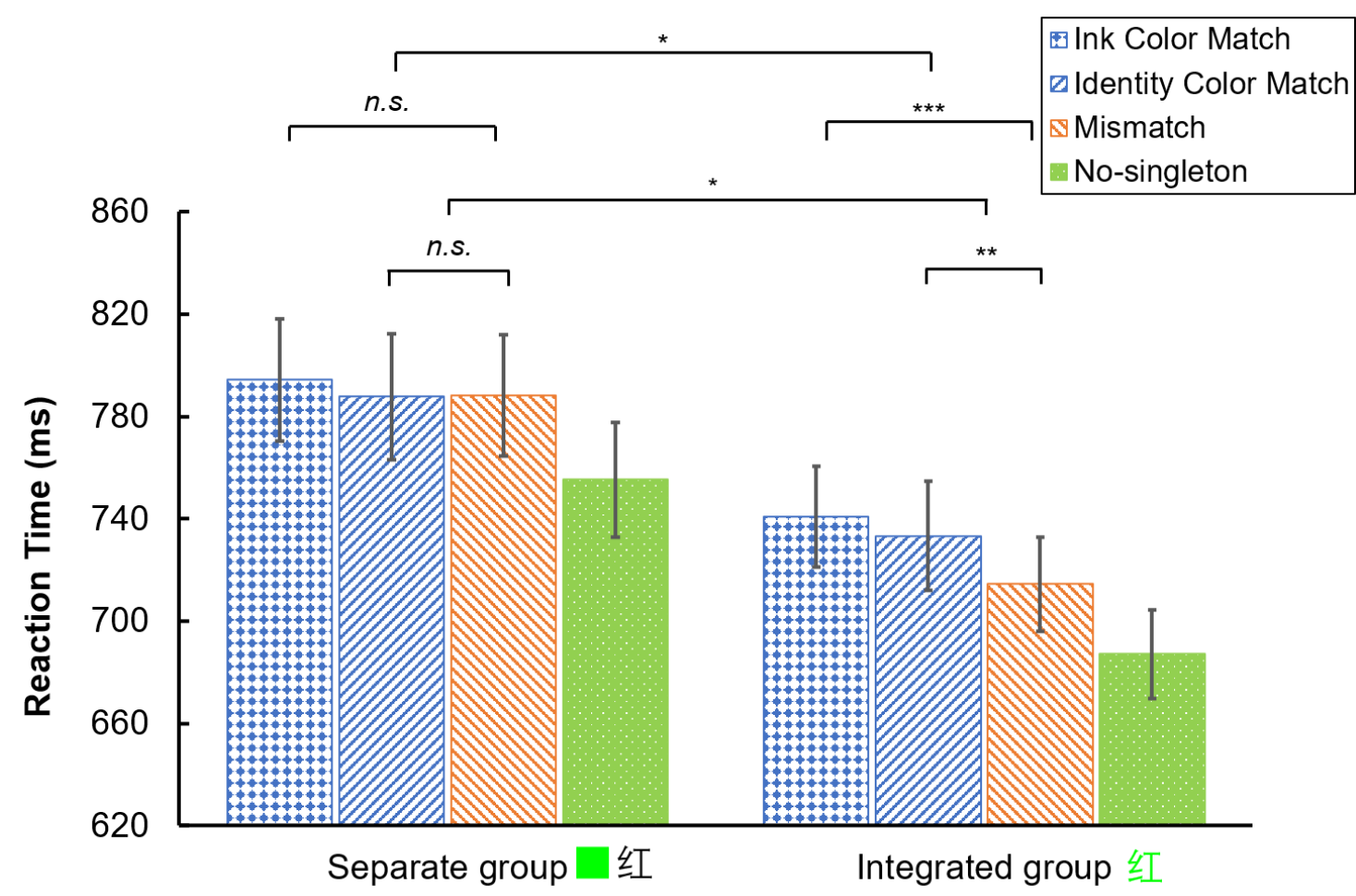

Figure 10. Mean search RT results for Experiment 5. $* p<.05$. $* * p<.01$. *** $p$ $<.001$. n.s. $=$ not significant. Error bars represent SEM.

Consistent with previous experiments, the results of Experiment 5 demonstrated that the effect of objecthood on attentional guidance could be generalized to two features from different modules.

\section{Discussion}

This study demonstrated that the unit of interaction between VWM and attention is an object, by providing converging evidence that the objecthood of multiple VWM representations significantly influences their ability for attentional guidance. Specifically, Experiments 1 and 2 showed that the VWM-driven attentional capture effect triggered by colors from an integrated object (i.e., a circle consisting of two or three different colored semicircles) was larger than that from two/three separate objects. 
Moreover, the magnitude of the attentional guidance effect of multiple color representations from the integrated object was almost equivalent to that of a singlecolor object. Experiment 3 demonstrated that such an attentional guidance difference was not caused by different memory fidelity. Experiments 4 and 5 extended these findings by generalizing the effect to two features from different dimensions (i.e., color and orientation), and from different modules (i.e., visual and verbal color representations).

These findings provide the first evidence indicating that the interaction between VWM and attention is implemented at the object level. In addition, as the object is the interactive unit between VWM and attention, the efficiency of the interaction should be mainly determined by the number of objects, instead of the number of features (even from the same dimension) or Boolean maps involved in each object. This consideration was supported by the current finding that attentional guidance was comparable between one integrated object consisting of multiple colors (two or three) and one object with a single color. However, it should be noted that the number of Boolean maps used in the current study is within the VWM capacity according to previous studies (Cowan, 2001; Luck \& Vogel, 1997). Thus, it is very likely that the interactive efficiency would be impaired if the number of Boolean maps, even from an integrated object, exceeds the VWM capacity. This question should be explored in future studies.

Notably, a recent study by Huang (2020) showed that the Boolean map, rather than the object, is the unit of VWM. Accordingly, two different colors would be represented as two distinct Boolean maps in VWM, regardless of whether they belong 
to an integrated object (e.g., circle), or two separate ones. The combination of this study with the current findings showed a very interesting distinction between the VWM storage and the interaction of VWM and visual attention, as the unit of VWM storage is Boolean map, while the unit of interaction is object. ${ }^{1}$ This distinction could be well reconciled when considering the organization of the representations in VWM. Take two colors case for example, we speculate that when two colors are held in VWM, not only the colors themselves are maintained, their constitute structure (i.e., came from same/different objects) is also stored. In other words, the VWM representation of the two colors consists of both color information and their constitute structure. This is in line with previous studies suggesting that the representations maintained in VWM depend on their perceptual format during the encoding stage, to some extent (Gao et.al., 2011; Serences et.al., 2009; Xu et al., 2020). VWM storage capacity is constrained by the colors information, and is independent of their constitute structure, as participants have to access two Boolean maps no matter when they come from the same or different objects in a VWM storage capacity task. In contrast, VWM-driven attentional-capture reflects an automatic attentional selection of information with a VWM color. The current results suggest that such a VWM-driven attentional selection process is constrained by the constitute structure of colors. This is somewhat in line with the previous work of Boolean map theory suggesting that the unit of attentional selection is an object, although the unit of access is a Boolean map (Huang, 2010).

\footnotetext{
${ }^{1}$ It should be noted that the present results are not in any conflict with the findings and claims of Boolean map theory, because previous work on Boolean map theory (e.g., Huang, 2020) is about the storage capacity of the VWM, and has not made any claim regarding the interaction between VWM and attention.
} 
This study has several important implications for the debate regarding the number of items in VWM simultaneously capable of guiding attention, as well as the architecture of the interaction between VWM and attention. First, our results demonstrate that attentional capture by VWM items is substantially influenced by the objecthood of item representations, which should be considered in future studies regarding the attentional capture ability for multiple items. Specifically, it may be more likely to observe the attentional capture effect for multiple items which could be easily grouped together. It should be noted that this does not mean that the grouping is the only factor that could influence the attentional capture by VWM items. In fact, Williams et al. (2019) found that the representational strength of memory items could also influence their ability to guide attention. However, this memory strength hypothesis can hardly explain our findings because our results showed that the memory strength of two different colors (probed by VWM performance such as accuracy or precision) was not beneficial when presenting them as an integrated object in our cases, as well as in some previous studies (e.g., Fougnie et al., 2010; Olson \& Jiang, 2002; Wheeler \& Treisman, 2002; Xu, 2002).

Furthermore, previous studies tried to address the debate by focusing mainly on assessing whether and when multiple items can simultaneously interact with attention (e.g., Bahle et al., 2019; Olivers, et al., 2011; van Moorselaar, et al., 2014; Williams et al., 2019). However, our study went beyond by exploring how to increase the interaction efficiency for multiple representations and demonstrated that grouping multiple colors into a single integrated object was an effective way to enhance their 
ability to guide attention. As the grouping could significantly affect the attentional guidance by multiple representations, it might lead to completely different conclusions if grouping strategy could be used in some experimental contexts while not in others. This indicates that more future studies should investigate the factors that determine the ability of attentional capture by multiple items, rather than concentrating on the debate itself.

Finally, our study firstly clarified the nature of the item in the interaction between VWM and attention as an object, regardless of the features or Boolean maps involved in it, an essential aspect for the resolution of the debate regarding their capacity of interaction. More broadly, this study also contributed to another related debate regarding the structure of VWM, that is, whether the capacity of the focus of attention (FoA) is limited to a single (McElree, 2006; Oberauer, 2002; Oberauer \& Bialkova, 2009), or multiple items (Cowan, 2001, 2005). Actually, apart from the memory-driven attentional guidance effect, previous studies have explored the capacity of FoA by multiple approaches. For example, researchers used spatially oriented visual cues to explore whether more than one item could be held in FoA (Matsukura \& Vecera, 2015). Similarly, with the reward cue, Allen \& Ueno (2018) investigated the extent to which attention can be strategically directed to multiple high-reward memory items. In these contexts, it is also crucial to first clarify the nature of the so-called "item" in FOA. If an "item" is equivalent to an object, our results seem to be consistent with the view that there is only one item with higher privilege status in the FOA, which is sufficient to drive the attentional guidance effect (Oberauer, 2002; Olivers et al., 2011). 
Nonetheless, we do not suggest that only one item/object can be held in the focus of attention; instead, it is possible that individuals can sometimes hold one or more objects in focus, especially when it is helpful for a task. As claimed by Oberauer and Bialkova (2009, 2011), this capacity limitation might actually reflect the function of FOA.

\section{Acknowledgements}

This work was supported by grants from National Science Foundation for

Distinguished Young Scholars of Zhejiang Province, China (No. LR19C090002), National Natural Science Foundation of China (No. 32171046) awarded to author Hui Chen.

\section{References}

Allen, R. J., \& Ueno, T. (2018). Multiple high-reward items can be prioritized in working memory but with greater vulnerability to interference. Attention, Perception \& Psychophysics, 80(7), 1731-1743. https://doi.org/10.3758/s13414-018-1543-6

Allen, R. J., Baddeley, A. D., \& Hitch, G. J. (2006). Is the binding of visual features in working memory resource-demanding? Journal of Experimental Psychology. General, 135(2), 298-313. https://doi.org/10.1037/0096-3445.135.2.298

Baddeley, A. D., \& Hitch, G. J. (1974). Working memory. In G. A. Bower (Ed.), The psychology of learning and motivation: Advances in research and theory (pp. 47-89). New York: Academic Press.

Baddeley, A. D., Allen, R. J., \& Hitch, G. J. (2011). Binding in visual working memory: The role of the episodic buffer. Neuropsychologia, 49(6), 13931400. https://doi.org/10.1016/j.neuropsychologia.2010.12.042

Bahle, B., Thayer, D. D., Mordkoff, J. T., \& Hollingworth, A. (2019). The architecture of working memory: Features from multiple remembered objects produce parallel, coactive guidance of attention in visual search. Journal of Experimental Psychology. General, 149(5), 967-983. https://doi.org/10.1037/xge0000694 
Beck, V. M., \& Vickery, T. J. (2019). Multiple states in visual working memory: Evidence from oculomotor capture by memory-matching distractors. Psychonomic Bulletin \&Review, 26(4), 1340-1346. https://doi.org/10.3758/s13423-019-01608-7

Cowan, N. (2001). The magical number 4 in short-term memory: A reconsideration of mental storage capacity. Behavioral and Brain Sciences, 24, 87-114. http://dx.doi.org/10.1017/S0140525X01003922

Cowan, N. (2005). Working memory capacity. New York, NY: Psychology Press.

Downing, P. E., \& Dodds, C. M. (2004). Competition in visual working memory for control of search. Visual Cognition, 11(6), 689-703. https://doi.org/10.1080/13506280344000446

Fougnie, D., Asplund, C. L., \& Marois, R. (2010). What are the units of storage in visual working memory? Journal of vision, 10(12), 27, 1-11. https://doi.org/10.1167/10.12.27

Frătescu, M., Van Moorselaar, D., \& Mathôt, S. (2019). Can you have multiple attentional templates? Large-scale replications of Van Moorselaar, Theeuwes, and Olivers (2014) and Hollingworth and Beck (2016). Attention, Perception, \& Psychophysics, 81(8), 2700-2709. https://doi.org/10.3758/s13414-019$\underline{01791-8}$

Fu, Y., Zhou,Y., Zhou, J., Shen, M., Chen, H. (In press). More attention with less working memory: The active inhibition of attended but outdated information. Science Advances

Gao, T., Gao, Z., Li, J., Sun, Z., \& Shen, M. (2011). The perceptual root of objectbased storage: an interactive model of perception and visual working memory. Journal of experimental psychology. Human perception and performance, 37(6), 1803-1823. https://doi.org/10.1037/a0025637

Gao, Z., Wu, F., Qiu, F., He, K., Yang, Y., \& Shen, M. (2016;2017;). Bindings in working memory: The role of object-based attention. Attention, Perception \& Psychophysics, 79(2), 533-552. https://doi.org/10.3758/s13414-016-1227-z

Grubert, A., \& Eimer, M. (2015). Rapid Parallel Attentional Target Selection in Single-Color and Multiple-Color Visual Search. Journal of Experimental Psychology: Human Perception and Performance, 41(1), 86-101. https://doi.org/10.1037/xhp0000019

Grubert, A., \& Eimer, M. (2016). All set, indeed! N2pc components reveal simultaneous attentional control settings for multiple target colors. Journal of Experimental Psychology: Human Perception and Performance, 42(8), 1215-1230. https://doi.org/10.1037/xhp0000221

Hollingworth, A., \& Beck, V. M. (2016). Memory-based attention capture when multiple items are maintained in visual working memory. Journal of Experimental Psychology. Human Perception and Performance, 42(7), 911917. https://doi.org/10.1037/xhp0000230 
Houtkamp, R., \& Roelfsema, P. R. (2006). The effect of items in working memory on the deployment of attention and the eyes during visual search. Journal of Experimental Psychology: Human Perception and Performance, 32(3), 423442. http://dx.doi.org/10.1037/0096-1523.32.2.423

Huang L. (2020). Unit of visual working memory: A Boolean map provides a better account than an object does. Journal of experimental psychology. General, 149(1), 1-30. https://doi.org/10.1037/xge0000616

Huang, L. (2010). What is the unit of visual attention? Object for selection, but Boolean map for access. Journal of Experimental Psychology: General, 139(1), 162-179. http://dx.doi.org/10.1037/a0018034

Huang, L., \& Pashler, H. (2007). A Boolean map theory of visual attention.

Psychological Review, 114(3), 599-631.

http://dx.doi.org/10.1037/0033295X.114.3.599

Huang, L., Treisman, A., \& Pashler, H. (2007). Characterizing the limits of human visual awareness. Science, 317(5839), 823-825.

http://dx.doi.org/10.1126/science.1143515

Langerock, N., Vergauwe, E., \& Barrouillet, P. (2014). The maintenance of crossdomain associations in the episodic buffer. Journal of Experimental Psychology. Learning, Memory, and Cognition, 40(4), 1096-1109. https://doi.org/10.1037/a0035783

Luck, S. J., \& Vogel, E. K. (1997). The capacity of visual working memory for features and conjunctions. Nature, 390(6657), 279-281. https://doi.org/10.1038/36846

Matsukura, M., \& Vecera, S. P. (2015). Selection of multiple cued items is possible during visual short-term memory maintenance. Attention, Perception \& Psychophysics, 77(5), 1625-1646. https://doi.org/10.3758/s13414-015-0836-2

McElree, B. (2006). Accessing recent events. In B. H. Ross (Ed.), Psychology of learning and motivation: Advances in research and theory (Vol. 46, pp. 155200). Sad Diego, CA: Elsevier. http://dx.doi.org/10.1016/s0079$\underline{7421(06) 46005-9}$

Oberauer K. (2002). Access to information in working memory: exploring the focus of attention. Journal of experimental psychology. Learning, memory, and cognition, 28(3), 411-421. http://dx.doi.org/10.1037/02787393.28.3.411

Oberauer, K., \& Bialkova, S. (2009). Accessing information in working memory: Can the focus of attention grasp two elements at the same time? Journal of Experimental Psychology: General, 138(1), 64-87. http://dx.doi.org/10.1037/a0014738

Oberauer, K., \& Bialkova, S. (2011). Serial and parallel processes in working memory after practice. Journal of Experimental Psychology:Human Perception and Performance, 37(2), 606-614. http://dx.doi.org/10.1037/a0020986

Olivers C. N. (2009). What drives memory-driven attentional capture? The effects of 
memory type, display type, and search type. Journal of experimental psychology. Human perception and performance, 35(5), 1275-1291. https://doi.org/10.1037/a0013896

Olivers, C. N. L., Meijer, F., \& Theeuwes, J. (2006). Feature-based memory-driven attentional capture: Visual working memory content affects visual attention. Journal of Experimental Psychology: Human Perception and Performance, 32(5), 1243-1265. https://doi.org/10.1037/0096-1523.32.5.1243

Olivers, C. N. L., Peters, J., Houtkamp, R., \& Roelfsema, P. R. (2011). Different states in visual working memory: When it guides attention and when it does not. Trends in Cognitive Sciences,15(7), 327-334. https://doi.org/10.1016/j.tics.2011.05.004

Olson, I. R., \& Jiang, Y. (2002). Is visual short-term memory object based? Rejection of the "strong-object" hypothesis. Perception \& Psychophysics, 64(7), 10551067. http://dx.doi.org/10.3758/BF03194756

Ort, E., \& Olivers, C. N. L. (2020). The capacity of multiple-target search. Visual Cognition, 28(5-8), 330-355. https://doi.org/10.1080/13506285.2020.1772430

Sasin, E., \& Fougnie, D. (2020). Memory-driven capture occurs for individual features of an object. Scientific Reports, 10(1), 19499-19499. https://doi.org/10.1038/s41598-020-76431-5

Serences, J. T., Ester, E. F., Vogel, E. K., \& Awh, E. (2009). Stimulus-specific delay activity in human primary visual cortex. Psychological Science, 20(2), 207214. https://doi.org/10.1111/j.1467-9280.2009.02276.x

Soto, D., Greene, C. M., Chaudhary, A., \& Rotshtein, P. (2012). Competition in working memory reduces frontal guidance of visual selection. Cerebral Cortex, 22(5), 1159-1169. https://doi.org/10.1093/cercor/bhr190

Soto, D., Heinke, D., Humphreys, G. W., \& Blanco, M. J. (2005). Early, involuntary top-down guidance of attention from working memory. Journal of Experimental Psychology: Human Perception and Performance, 31(2), 248261. https://doi.org/10.1037/0096-1523.31.2.248

Sun, S. Z., Shen, J., Shaw, M., Cant, J. S., \& Ferber, S. (2015). Automatic capture of attention by conceptually generated working memory templates. Attention, perception \& psychophysics, 77(6), 1841-1847.

https://doi.org/10.3758/s13414-015-0918-1

van Moorselaar, D., Theeuwes, J., \& Olivers, C. N. L. (2014). In competition for the attentional template: Can multiple item within visual working memory guide attention? Journal of Experimental Psychology. Human Perception and Performance, 40(4), 1450-1464. https://doi.org/10.1037/a0036229

Wheeler, M. E., \& Treisman, A. M. (2002). Binding in short-term visual memory. Journal of Experimental Psychology. General, 131(1), 48-64. https://doi.org/10.1037/0096-3445.131.1.48 
Williams, J., Brady, T.F., and Störmer, V.S. (2019). Natural variation in the fidelity of working memory representations cause only one item to guide attention. PsyArXiv. Preprint. https://doi.org/10.31234/osf.io/c4t92

Xu, M., Fu, Y., Yu, J., Zhu, P., Shen, M., \& Chen, H. (2020). Source information is inherently linked to working memory representation for auditory but not for visual stimuli. Cognition, 197(2020), 104160.

https://doi.org/10.1016/j.cognition.2019.104160

$\mathrm{Xu}, \mathrm{Y}$. (2002). Limitations of object-based feature encoding in visual short-term memory. Journal of Experimental Psychology: Human Perception and Performance, 28(2), 458-468. http://dx.doi.org/10.1037/00961523.28.2.458

Zhou, C., Lorist, M. M., \& Mathôt, S. (2020). Concurrent guidance of attention by multiple working memory items: Behavioral and computational evidence. Attention, Perception \& Psychophysics, 82(6), 2950-2962. https://doi.org/10.3758/s13414-020-02048-5 